\title{
The Influence of a KDT501, a Novel Isohumulone, on Adipocyte Function in Humans
}

\author{
Brian S. Finlin', Beibei Zhu', Bernard P. Kok' ${ }^{2}$, Cristina Godio' ${ }^{2}$, Philip M. Westgate ${ }^{3}$, \\ Neile Grayson ${ }^{4}$, Robert Sims ${ }^{4}$, Jeffrey S. Bland ${ }^{4}$, Enrique Saez ${ }^{2}$ and Philip A. Kern ${ }^{1 *}$ \\ ${ }^{1}$ The Department of Internal Medicine, Division of Endocrinology, The Barnstable Brown Diabetes and Obesity Center, \\ University of Kentucky, Lexington, KY, United States, ${ }^{2}$ Department of Molecular Medicine, Scripps Research Institute, \\ La Jolla, CA, United States, ${ }^{3}$ College of Public Health, University of Kentucky, Lexington, KY, United States, \\ ${ }^{4}$ Kindex Pharmaceuticals, Seattle, WA, United States
}

Objective: In a phase II clinical trial in nine obese, insulin-resistant humans, we observed that treatment with KDT501, a novel isohumulone drug, increased total and high-molecular weight $(\mathrm{HMW})$ adiponectin in plasma. The objective was to determine whether KDT501 increased adiponectin secretion from subcutaneous white adipose tissue (SC WAT) and the underlying mechanism(s).

OPEN ACCESS

Edited by:

Jan Polák,

Charles University, Czechia

Reviewed by: Alberto Camacho,

Autonomous University of Nuevo Leon, Mexico

Michaela Tencerova,

University of Southern Denmark

Odense, Denmark

*Correspondence:

Philip A. Kern

philipkern@uky.edu

Specialty section:

This article was submitted

to Diabetes,

a section of the journa

Frontiers in Endocrinology

Received: 20 July 2017 Accepted: 15 September 2017 Published: 29 September 2017

Citation:

Finlin BS, Zhu B, Kok BP, Godio C, Westgate PM, Grayson N, Sims R, Bland JS, Saez E and Kern PA (2017)

The Influence of a KDT501,

a Novel Isohumulone, on

Adipocyte Function in Humans.

Front. Endocrinol. 8:255.

doi: 10.3389/fendo.2017.00255
Methods: Nine obese participants with either prediabetes or with normal glucose tolerance plus three features of metabolic syndrome were part of the study. SC WAT biopsies were performed before and after 28 days of KDT501 treatment in a clinical research setting. In addition, a cold stimulus was used to induce thermogenic gene expression. Adiponectin secretion was measured, and gene expression of 130 genes involved in adipose tissue function was determined. The effect of KDT501 on adipocyte mitochondrial function was analyzed in vitro.

Results: SC WAT explants secreted more total and HMW adiponectin after KDT501 treatment $(P<0.05)$. After KDT501 treatment, a number of genes involved in thermogenesis and lipolysis were induced by cold $(P<0.05)$. KDT501 also potentiated $\beta$-adrenergic signaling $(P<0.001)$ and enhanced mitochondrial function in adipocytes $(P<0.001)$.

Conclusion: KDT501 induced adiponectin secretion posttranscriptionally and increased gene expression of thermogenic and lipolytic genes in response to cold stimulation. These beneficial effects on SC WAT may be explained by the ability of KDT501 to potentiate $\beta$-adrenergic signaling and enhance mitochondrial function in adipocytes.

Clinical Trial Registration: https://www.ClinicalTrials.gov, ID number: NCT02444910.

Keywords: adiponectin, adipocyte secretion, adipose tissue biology, gene expression profiling, metabolic syndrome, mitochondria

\section{INTRODUCTION}

KDT501 is a compound chemically derived from hops that has antidiabetic effects in rodents (1). More specifically, KDT501 is the potassium salt of the $n$-(isobutyl) congener of a tetrahydro iso-alpha acid, also known as isohumulone. We have recently analyzed the effect of 1 month of KDT501 treatment in nine obese, insulin-resistant subjects in an open-label, phase II clinical trial (2). Although 
oral glucose tolerance did not improve significantly, there were improvements in several secondary outcomes. KDT501 treatment reduced plasma triglyceride levels during a lipid tolerance test. Total and high-molecular weight (HMW) adiponectin were higher in plasma after KDT501 treatment (2). Adiponectin is an anti-inflammatory, insulin-sensitizing adipokine that is negatively correlated with several aspects of metabolic syndrome and polycystic ovary syndrome (PCOS) (3) and acts at multiple tissues to promote metabolic homeostasis [recently reviewed in Ref. (4)]. Consistent with this, plasma TNF $\alpha$ was reduced by KDT501 treatment (2).

Adiponectin is specifically expressed by adipocytes, suggesting that KDT501 treatment in this clinical trial affected adipose tissue to increase plasma adiponectin levels (2). KDT501 has pleiotropic effects on gene expression in vitro. For instance, KDT501 promoted adiponectin gene expression in a human adipocyte primary cell culture model (1). KDT501 also inhibited inflammatory gene expression in macrophages in vitro (1). In obesity, adipose tissue secretes $\operatorname{TNF} \alpha(5,6)$, an inflammatory cytokine that is inversely coordinated with adiponectin expression and insulin sensitivity (7). These observations suggest that KDT501 treatment reduces some measures of adipose dysfunction in part by remodeling gene transcription.

In this study, we focused on adipose tissue to identify mechanisms by which KDT501 functions in vivo in these nine obese human research participants. We evaluated adiponectin secretion from abdominal subcutaneous white adipose tissue (SC WAT) explant secretions of the participants before and after treatment with KDT501 from our phase 2 study (2). We then comprehensively evaluated the subcutaneous adipose tissue transcriptional response to KDT501. Since KDT501 promotes weight loss in rodents (1), we hypothesized that KDT501 treatment would reduce adipose dysfunction that occurs with obesity in humans. Comprehensive analysis of gene expression could thus provide insight into the mechanism by which KDT501 increases adiponectin secretion in vivo and affects overall SC WAT function. The panel of genes in our multiplex assay included genes involved in the function of adipose tissue and genes involved in adipose dysfunction such as inflammatory cytokines, immune cell type markers, and fibrosis genes. Because KDT501 was found to stimulate thermogenic genes in cultured human adipocytes (1), we measured these genes before and after a brief cold stimulus. To gain mechanistic insight into the effect of KDT501 on adipocytes, we evaluated the effect of KDT501 on adipocyte mitochondrial bioenergetics and fatty acid oxidation in vitro.

\section{MATERIALS AND METHODS}

\section{Human Subjects and Study Design}

All subjects gave informed consent, and the protocols were approved by the Institutional Review Board at the University of Kentucky. Recruitment for this study was through local advertising and initial phone screening eliminated from consideration participants who could not participate or who did not meet inclusion criteria, such as BMI and age. 15 potential subjects were invited for in-person screening, which included an OGTT and fasting labs, and 9 of these subjects met inclusion/exclusion criteria and were enrolled in the study. All nine of these subjects completed the study. The characteristics of these subjects; inclusion and exclusion criteria; and the Phase II KDT501 drug trial (ClinicalTrials.gov ID number NCT02444910) have recently been reported (2). In brief, nine obese, non-diabetic, insulin-resistant subjects were given KDT501 in escalating doses for 28 days during the summer. The relatively short duration of treatment and the limited doses of KDT501 was a requirement of the FDA during this phase 2A study. This short-term treatment did not cause a change in appetite, weight (pre: $100.8 \pm 5.5 \mathrm{~kg}$; post: $100.3 \pm 5.7 \mathrm{~kg} ; P=0.63$ ), \% body fat (pre: $47.5 \pm 1.55$; post: $47.6 \pm 1.5 ; P=0.5$ ), resting energy expenditure (pre: $1,725 \pm 79 \mathrm{kcal} /$ day; post: $1,670 \pm 106 \mathrm{kcal} /$ day; $P=0.29)$, or respiratory quotient (pre: $0.86 \pm 0.02$; post: $0.87 \pm 0.02 ; P=0.0 .86$ ); all values are mean \pm SEM. SC WAT biopsies were performed before and after 28 days of KDT501 treatment; all posttreatment biopsies were obtained between 0 and 3 days after the last dose of KDT501. In addition, a cold stimulus was used to determine whether KDT501 affected thermogenic gene induction. A subcutaneous adipose biopsy was performed on the right side of the abdomen. An ice pack was applied to the left side of the abdomen for $30 \mathrm{~min}$, and a second biopsy was performed $4 \mathrm{~h}$ later on the cold-stimulated adipose. A portion of the adipose tissue was analyzed for secretion as described below. The remaining adipose tissue was immediately frozen in liquid nitrogen and stored at $-80^{\circ} \mathrm{C}$.

\section{Adipose Tissue Explant Secretions}

We weighed $0.5 \mathrm{~g}$ of fat from each SC WAT biopsy, placed the explant into $2 \mathrm{~mL}$ Dulbecco's modified Eagles' medium (DMEM), and incubated for $1 \mathrm{~h}$ at $37^{\circ} \mathrm{C}$. The adipose explant was removed, and the DMEM was centrifuged to remove debris. The experiment thus maintained the SC WAT weight to volume of DMEM ratio similar to the normalization applied by Kovacova and colleagues (8). The medium was diluted 1:100 and analyzed with a total and HMW adiponectin ELISA kit (47-ADPHU-E01; Alpco, Salem, NH, USA) according to the manufacturer's instructions.

\section{Gene Expression}

RNA was prepared from the subcutaneous adipose tissue using a Qiagen RNAeasy Lipid Tissue Mini kit (Qiagen, Valencia, CA, USA). RNA quantity and quality were determined using an Agilent 2100 Bioanalyzer (Palo Alto, CA, USA). The expression of 130 genes was analyzed using the Nanostring nCounter multiplex system (gene names and accession numbers are in Table S1 in Supplementary Material). The data were normalized to the positive controls and the geometric mean of six housekeeping genes (Table S1 in Supplementary Material) according to the manufacturer's instructions. The background level of the standard negative controls supplied by Nanostring was very low and not subtracted. We also determined the negative control value of the individual probes by doing no RNA (water) controls. These values, which are the average of three controls, were also low (see Tables S2-S5 in Supplementary Material). The gene expression of ERp44, ERo1-La, and DsbA-L was determined by real-time 
reverse transcriptase polymerase chain reaction (RT-PCR) as described (9) and normalized the geometric mean of the same six genes utilized in the Nanostring assay (Table S1 in Supplementary Material).

\section{Cell Culture}

Primary brown preadipocytes were isolated from neonatal wild-type C57BL/6 pups as previously described (10). Briefly, brown adipose tissue was digested for $30 \mathrm{~min}$ at $37^{\circ} \mathrm{C}$ using $1.5 \mathrm{mg} / \mathrm{mL}$ collagenase type I (Worthington) in PBS containing the following additional components: $62 \mathrm{mM} \mathrm{NaCl}, 2.5 \mathrm{mM}$ $\mathrm{KCl}, 0.65 \mathrm{mM} \mathrm{CaCl}_{2}, 2.5 \mathrm{mM}$ glucose, $50 \mathrm{mM}$ HEPES, and $2 \%$ fatty acid-free BSA. Cells were strained using a $100 \mu \mathrm{M}$ filter, plated, and grown to confluence in 20\% FBS-DMEM. Differentiation was initiated by replacing the growth media with 10\% FBS-DMEM supplemented with $172 \mathrm{nM}$ insulin, $1 \mu \mathrm{M}$ dexamethasone, $0.5 \mathrm{mM}$ 3-isobutyl-1-methylxanthine (IBMX), $1 \mathrm{nM}$ triiodothyronine (T3), and $1 \mu \mathrm{M}$ rosiglitazone. After 2 days, adipogenesis induction media were replaced with $10 \%$ FBS-DMEM containing $172 \mathrm{nM}$ insulin and $1 \mathrm{nM} \mathrm{T} 3$ and refreshed every other day. Five days after the induction of differentiation, differentiated brown adipocytes were replated onto a 96-well Seahorse plate at a density of 5,000 cell/well and allowed to settle overnight. Cells were treated with vehicle, rosiglitazone, and KDT501 for $16 \mathrm{~h}$ before analysis of mitochondrial respiration parameters on a Seahorse XFe96 extracellular flux analyzer. 3T3-L1 preadipocytes were cultured and differentiated as previously described (11). Briefly, 3T3-L1 preadipocytes were maintained in 10\% BCS-DMEM. To induce differentiation, cells were replated and allowed to reach confluence. Two days post-confluence, cells were treated with $172 \mathrm{nM}$ insulin, $1 \mu \mathrm{M}$ dexamethasone, $0.5 \mathrm{mM}$ IBMX, and $1 \mu \mathrm{M}$ rosiglitazone in 10\% FBS-DMEM. After 2 days, adipogenesis induction media were replaced with $10 \%$ FBS-DMEM supplemented with $172 \mathrm{nM}$ insulin. Differentiated adipocytes were replated onto 96-well Seahorse plates at 6,000 cells per well and allowed to settle overnight. Cells were treated with vehicle, rosiglitazone, or KDT501 for $48 \mathrm{~h}$ before the fatty acid oxidation rate was measured in the Seahorse XFe96.

\section{Mitochondrial Bioenergetics and Fatty Acid Oxidation Rate Assays}

Analyses of mitochondrial respiration parameters and fatty acid oxidation rates were performed in an XFe6 instrument following the manufacturer's instructions (Seahorse Bioscience). Briefly, primary brown adipocytes were switched to substrate-free DMEM supplemented with $10 \mathrm{mM}$ glucose, $10 \mathrm{mM}$ pyruvate, 1× Glutamax, $10 \mathrm{mM}$ HEPES $\mathrm{pH} 7.4$, and a Mitostress test was performed. The oxygen consumption rate was measured in real time to document basal respiration and four sequential injections were then performed: (1) $100 \mathrm{nM}$ norepinephrine (NE); (2) $2 \mu \mathrm{M}$ oligomycin; (3) $1 \mu \mathrm{M}$ carbonyl cyanide-4-(trifluoromethoxy) phenylhydrazone (FCCP); and (4) a combination of $2 \mu \mathrm{M}$ rotenone and $2 \mu \mathrm{M}$ antimycin $\mathrm{A}$. To measure the fatty acid oxidation rate, 3T3-L1 adipocytes were switched to assay media (KrebsHenseleit Buffer, $2.5 \mathrm{mM}$ final glucose, $0.5 \mathrm{mM}$ carnitine, and
5 mM HEPES; $\mathrm{pH}$ 7.4) for $1 \mathrm{~h}$ before the addition of vehicle (33 $\mu \mathrm{M}$ BSA) or $167 \mu \mathrm{M}$ palmitate in complex with BSA (5:1). Real-time oxygen consumption was measured immediately after palmitate addition and following injections of $4 \mu \mathrm{M}$ oligomycin, $4 \mu \mathrm{M}$ FCCP, and a combination of $2 \mu \mathrm{M}$ rotenone and $2 \mu \mathrm{M}$ antimycin A. The concentration of oligomycin and FCCP was increased in fatty acid oxidation rate assays to account for the presence of BSA.

\section{Statistics}

Unless noted, data were analyzed with a two-tailed, paired $t$-test. To identify genes that had a differential response to cold stimulus by KDT501 treatment, we calculated the difference in the response to cold stimulus before and after KDT501 treatment and then performed a two-sample $t$-test on this difference. Statistical significance was set at $P<0.05$. Data from in vitro experiments with cultured adipocytes were analyzed by one-way analysis of variance (ANOVA).

\section{RESULTS}

\section{KDT501 Treatment Stimulates Adiponectin Secretion}

We previously reported that KDT501 treatment increased total and HMW adiponectin levels in plasma (2). When the SC WAT biopsies were performed in that study, a portion was immediately frozen for mRNA analyses and another portion (0.5 g) was placed in DMEM for $1 \mathrm{~h}$ so that we could analyze secreted proteins. We evaluated total and HMW adiponectin secretion from SC WAT explants before and after KDT501 treatment. KDT501 treatment induced a 1.5-fold increase in the secretion of total adiponectin (Figure 1A; $P<0.05$ ) and HMW adiponectin (Figure 1B; $P<0.05$ ) from the adipose tissue explants. However, adiponectin gene expression was unchanged by KDT501 treatment (Figure 1C), indicating that the increase in secretion occurs by a posttranscriptional mechanism. These results indicate that increased secretion of adiponectin from subcutaneous adipose tissue contributes to the increase in plasma adiponectin of these research participants after KDT501 treatment (2). Linear regression modeling was used to assess associations between changes in adiponectin secretion by KDT501 treatment and gender, age, and BMI. However, results were not statistically significant and therefore are not presented.

\section{Effect of KDT501 on Adipose Tissue Gene Expression}

To identify potential mechanisms of action of KDT501, we analyzed gene expression in abdominal SC WAT with the Nanostring nCounter multiplex system. The gene panel comprised cytokines, chemotactic factors, immune cell markers, fibrosis markers, angiogenesis markers, genes involved in lipid handling, adipogenesis makers, genes involved in thermogenesis, and markers of brown and beige adipose (Table S1 in Supplementary Material). Table 1 shows that KDT501 treatment caused significant changes in only four adipose genes; four additional genes with non-significant trends $(0.05<P<0.1)$ for 


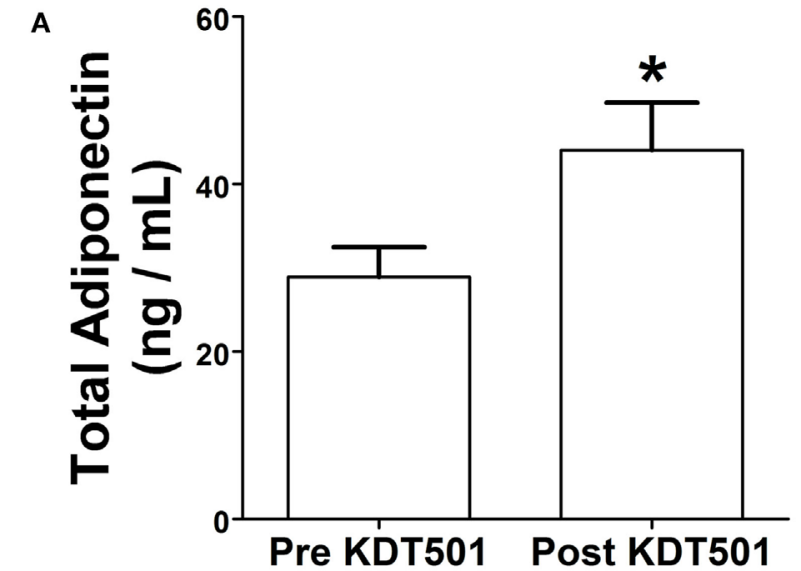

B
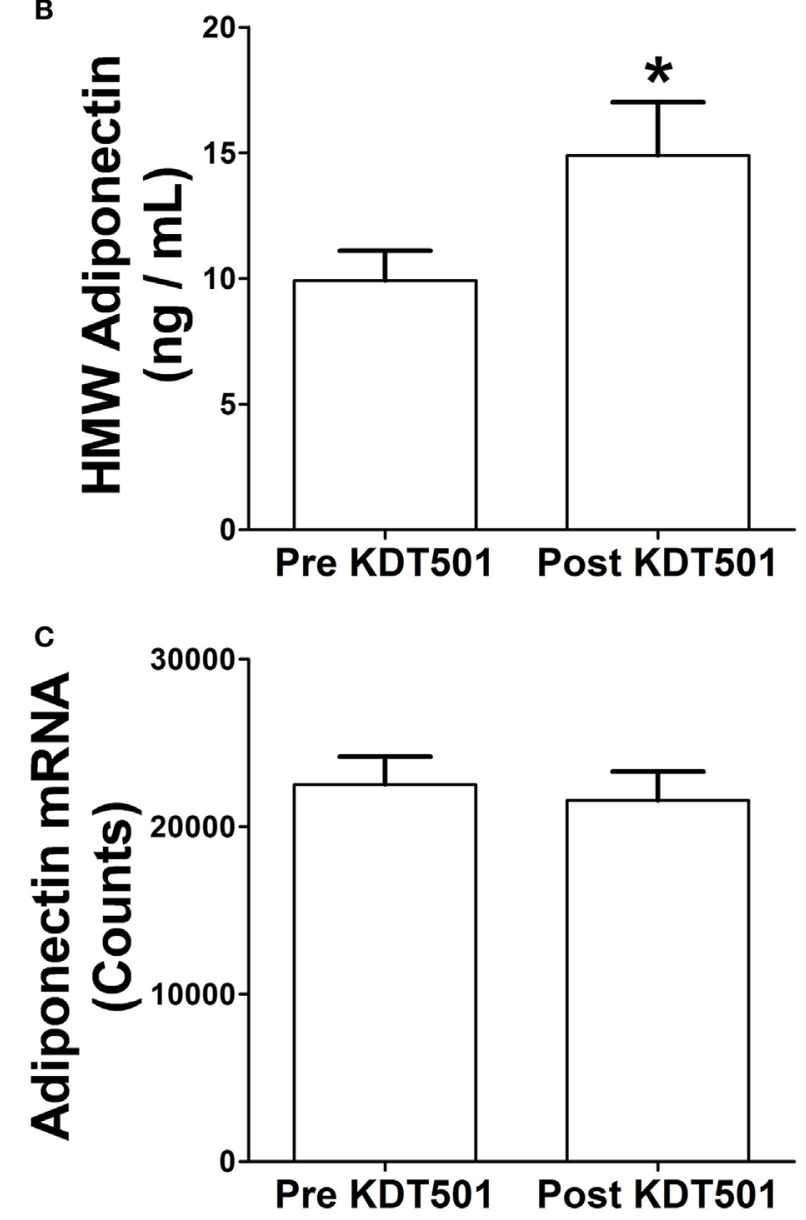

FIGURE 1 | KDT501 induces total and high-molecular weight (HMW) adiponectin secretion by adipose tissue explants from obese, insulin-resistant subjects. Adipose tissue explants obtained before and after KDT501 treatment were incubated in Dulbecco's modified Eagles' medium (DMEM) for $1 \mathrm{~h}$ at $37^{\circ} \mathrm{C}$. (A) Total and (B) HMW adiponectin concentrations in the DMEM were measured by ELISA and are expressed as the concentration/g adipose/h. (C) Adiponectin gene expression was measured in the corresponding adipose tissue biopsy with the Nanostring nCounter system as described in the Section "Materials and Methods." Data represent the mean $\pm \operatorname{SEM}(n=9)$; data in all panels were analyzed by a paired, two-tailed Student's $t$-test $\left({ }^{*} P<0.05\right)$.
TABLE 1 | Gene expression changed by KDT501 treatment.

\begin{tabular}{lcccc}
\hline Gene $^{\text {a }}$ & Pre KDT501 & Post KDT501 & Fold change & $\boldsymbol{P}$ \\
\cline { 4 - 4 } & & & Post/pre & \\
\hline TNFRSF12A & $12 \pm 2$ & $16 \pm 2$ & 1.345 & 0.037 \\
ACACA & $229 \pm 45$ & $196 \pm 35$ & 0.857 & 0.038 \\
MPZL2 & $52 \pm 8$ & $73 \pm 12$ & 1.407 & 0.041 \\
DGAT2 & $12,059 \pm 2,187$ & $10,482 \pm 1,941$ & 0.869 & 0.043 \\
LPL & $17,455 \pm 2,836$ & $15,793 \pm 2,584$ & 0.905 & 0.068 \\
TMEM26 & $16 \pm 2$ & $13 \pm 2$ & 0.856 & 0.072 \\
TIMP1 & $1,382 \pm 89$ & $1,600 \pm 158$ & 1.157 & 0.075 \\
DIO2 & $27 \pm 6$ & $38 \pm 9$ & 1.392 & 0.095 \\
\hline
\end{tabular}

aSCWAT was isolated before and after KDT501 treatment, and gene expression determined with the Nanostring nCounter system as described in the Section "Materials and Methods."

The data are presented as means (nCounter Counts) \pm SEM ( $n=9$; two-tailed, paired Student's t-test).

change are also indicated in Table 1. KDT501 treatment reduced the mRNA expression of ACACA (Table 1; 0.86-fold; $P=0.038$ ), which regulates fatty acid synthesis, and DGAT, which regulates triglyceride formation (Table $1 ; 0.87$-fold; $P=0.043$ ). The mRNA expression of LPL, which regulates lipid and lipoprotein uptake, tended to be reduced (Table $1 ; P=0.068$ ). Together, these results suggest a slight reduction in genes controlling adipose tissue lipid synthesis, uptake, and storage. MPZL2, which is a brown adipose marker (12), was induced 1.4-fold (Table $1 ; P=0.041$ ), and there was a trend for an increase in DIO2, which is involved in energy metabolism through the conversion of T4 into T3. However, UCP1 $(P=0.7)$ and PGC1 $\alpha(P=0.37)$, genes that are characteristic of brown and beige adipocytes, were not changed (Table S2 in Supplementary Material). The significance of the increases in MPZL2 and TNFRSF12A (Fn14, which is involved in many cell processes, especially involving tissue injury) is not known. Although KDT501 has anti-inflammatory effects in vitro, KDT501 treatment did not significantly reduce $\mathrm{TNF} \alpha$, IL12 $\beta$, IL1 $\beta$, or other inflammatory cytokines and immune cell markers in the multiplex assay panel (Table S2 in Supplementary Material). Finally, KDT501 did not change leptin gene expression (Table S2 in Supplementary Material). The data for all of the genes measured and the negative control values for each probe are in Table S2 in Supplementary Material.

\section{KDT501 Treatment Alters the Response of Adipose Tissue to Cold Stimulation}

KDT501 promotes weight loss in mice, and microarray results from human adipocytes indicate that KDT501 stimulates thermogenic pathways in adipocytes (1), suggesting that KDT501 may promote WAT beiging. We have previously reported that a 30-min cold exposure stimulates beiging pathways in the adipose tissue of lean humans (13). Therefore, before and after KDT501 treatment, we applied an ice pack to one side of the abdomen for $30 \mathrm{~min}$ and $4 \mathrm{~h}$ later performed an adipose tissue biopsy, as described in the Section "Materials and Methods." We then determined whether this cold stimulus changed gene expression and whether KDT501 treatment altered the response to cold. Before KDT501 treatment, the application of cold resulted in 
the significant repression of four unrelated genes, SCD, MMP14, AGTR2, and IL18 (Table 2; data for all of the remaining genes are in Table S3 in Supplementary Material).

After KDT501 treatment, a number of genes involved in lipolysis and lipid catabolism were significantly induced by cold (Table 3). These included PPAR $\alpha$, PGC1 $\alpha$, HSL, and ANGPTL4 (Table 3). Adipose triglyceride lipase (PNPLA2) and the coactivator of ATGL (CGI-58; ABHD5) showed a trend for induction (Table 3). VEGF, which stimulates angiogenesis and beiging (14), was also induced by icing (Table 3). Despite the induction

TABLE 2 | Gene expression changed by cold before KDT501 treatment.

\begin{tabular}{lcccc}
\hline Gene & Pre cold & Post cold & Fold change & $P$ \\
& & & Post/pre & \\
\hline SCD & $49,210 \pm 12,496$ & $44,454 \pm 12,115$ & 0.90 & 0.008 \\
AGTR2 & $7 \pm 1$ & $4 \pm 1$ & 0.66 & 0.013 \\
MMP14 & $548 \pm 40$ & $499 \pm 34$ & 0.91 & 0.030 \\
IL18 & $88 \pm 10$ & $74 \pm 5$ & 0.83 & 0.034 \\
FABP1 & $10 \pm 2$ & $5 \pm 1$ & 0.51 & 0.055 \\
ACLY & $2,998 \pm 624$ & $2,490 \pm 444$ & 0.83 & 0.061 \\
ADRB2 & $72 \pm 4$ & $66 \pm 4$ & 0.92 & 0.066 \\
CD163 & $1,074 \pm 84$ & $978 \pm 71$ & 0.91 & 0.069 \\
THBS1 & $968 \pm 125$ & $1,163 \pm 149$ & 1.20 & 0.079 \\
RBP4 & $23,669 \pm 1,752$ & $21,269 \pm 2,232$ & 0.90 & 0.091 \\
CCL24 & $90 \pm 21$ & $75 \pm 16$ & 0.83 & 0.094 \\
ADIPOQ & $22,520 \pm 1,671$ & $20,773 \pm 1,250$ & 0.92 & 0.100
\end{tabular}

aSCWAT was isolated before and after the cold stimulus before KDT501 treatment, and gene expression determined with the Nanostring nCounter system as described in the Section "Materials and Methods."

The data are presented as means ( $n$ Counter Counts) \pm SEM ( $n=9$; two-tailed, paired Student's t-test)

TABLE 3 | Gene expression changed by cold after KDT501 treatment.

\begin{tabular}{lcccc}
\hline Gene & Pre cold & Post cold & Fold change & P \\
\cline { 3 - 4 } & & & \\
& & & Post/pre & \\
\hline ANGPTL4 & $1,136 \pm 174$ & $1,659 \pm 163$ & 1.46 & 0.001 \\
CCL1 & $7 \pm 1$ & $12 \pm 1$ & 1.59 & 0.003 \\
BCL2 & $456 \pm 35$ & $543 \pm 45$ & 1.19 & 0.004 \\
CSF2 & $46 \pm 4$ & $53 \pm 3$ & 1.17 & 0.009 \\
LIPE & $225 \pm 27$ & $245 \pm 26$ & 1.09 & 0.018 \\
CEBPA & $6,498 \pm 568$ & $7,549 \pm 504$ & 1.16 & 0.019 \\
PPARA & $179 \pm 13$ & $192 \pm 12$ & 1.07 & 0.030 \\
VEGFA & $1,013 \pm 86$ & $1,150 \pm 75$ & 1.13 & 0.035 \\
PPARGC1A & $48 \pm 6$ & $57 \pm 9$ & 1.19 & 0.038 \\
DIO2 & $41 \pm 9$ & $24 \pm 5$ & 0.58 & 0.049 \\
TEK & $263 \pm 18$ & $305 \pm 24$ & 1.16 & 0.051 \\
MMP9 & $311 \pm 117$ & $210 \pm 42$ & 0.68 & 0.052 \\
ABHD5 & $537 \pm 65$ & $597 \pm 74$ & 1.11 & 0.054 \\
FABP3 & $219 \pm 61$ & $148 \pm 13$ & 0.68 & 0.065 \\
ITGAM & $291 \pm 27$ & $256 \pm 19$ & 0.88 & 0.068 \\
ANGPTL1 & $288 \pm 27$ & $222 \pm 14$ & 0.77 & 0.075 \\
PNPLA2 & $6,664 \pm 648$ & $7,129 \pm 490$ & 1.07 & 0.078 \\
LPL & $16,496 \pm 2,584$ & $18,240 \pm 2,305$ & 1.11 & 0.090 \\
FGF2 & $1,019 \pm 107$ & $120 \pm 91$ & 1.10 & 0.092 \\
\hline & & & &
\end{tabular}

aSCWAT was isolated before and after the cold stimulus after KDT501 treatment, and gene expression was determined with the Nanostring nCounter system as described in the Section "Materials and Methods."

The data are presented as means ( $n$ Counter Counts) \pm SEM $(n=9$; two-tailed, paired Student's t-test). of lipolytic genes and PGC1 $\alpha$ by cold, the 1.2 -fold increase in UCP1 was not significant $(P=0.34$; Table S4 in Supplementary Material). CCL1, a chemokine for eosinophils and Th2 T cells $(15,16)$, was induced by cold after KDT501, which could be important given the role of type 2 immunity (e.g., IL4 and IL-13) in adipose beiging (17-20). Data for the remaining genes are in Table S4 in Supplementary Material.

We identified genes that had a differential response to cold before and after KDT501 treatment as described in methods (Table S5 in Supplementary Material). This analysis identified many of the same genes in Table 3 and some additional genes, providing further insight. Before KDT501 treatment, the gene expression of the thermogenic genes PGC1 $\alpha$, TMEM26, and PPAR $\alpha$ decreased slightly after cold stimulation; after KDT501 treatment, the expression of these genes increased (Figures 2A-C; $P<0.05)$. VEGF, which induces beiging (14) and angiogenesis, was also increased by cold after KDT501 treatment (Figure 2D; $P<0.01)$. PAI-1 is regulated by TGF $\beta$ signaling, which inhibits PGC1 $\alpha$ and energy expenditure (21); PAI- 1 was increased by cold before KDT501, but did not increase after KDT 501 (Figure 2E; $P<0.05)$. Overall, this suggests that KDT501 promotes the expression of genes involved in lipolysis and fat catabolism in response to cold. In addition, LPL increased in response to cold after KDT501 treatment (Figure 2F).

\section{KDT501 Enhances $\beta$-Adrenergic Responses and Fatty Acid Oxidation in Adipocytes}

A potential explanation for the observation that KDT501 treatment enhances expression of thermogenic genes in response to cold is that KDT501 sensitizes adipocytes to $\beta$-adrenergic stimuli. To directly test this notion, fully differentiated mouse primary brown adipocytes were pretreated with KDT501 or rosiglitazone for $16 \mathrm{~h}$, and their response to acute exposure to $\mathrm{NE}$ was then monitored in real time by measuring the rate of oxygen consumption in a Seahorse XFe instrument. As expected for brown adipocytes, NE exposure stimulated oxygen consumption in vehicle (DMSO)-treated cells (Figure 3A). Pretreatment with KDT501 robustly enhanced the response to NE (Figure 3A; KDT501 $P<0.001$; rosiglitazone $P<0.001)$. Pretreatment with the positive control rosiglitazone had a similar effect. To expand these observations and determine the extent to which KDT501 treatment could also induce a more oxidative state in white adipocytes, we treated fully differentiated 3T3-L1 adipocytes with KDT501 for $48 \mathrm{~h}$ and determined the rate of fatty acid oxidation. KDT501 treatment significantly increased the endogenous, or basal $(P<0.001)$, and the maximal $(P<0.001)$ rate of exogenous fatty acid oxidation (Figure 3B), suggesting that KDT501 treatment boosts mitochondrial function. Together, these data show that KDT501 has direct effects on adipocytes, potentiating $\beta$-adrenergic signaling and fatty acid oxidation.

\section{Cold Treatment Stimulates Adiponectin Secretion}

The analysis of gene expression and in vitro assays above suggest that KDT501 enhances $\beta$-adrenergic responses of adipocytes. 

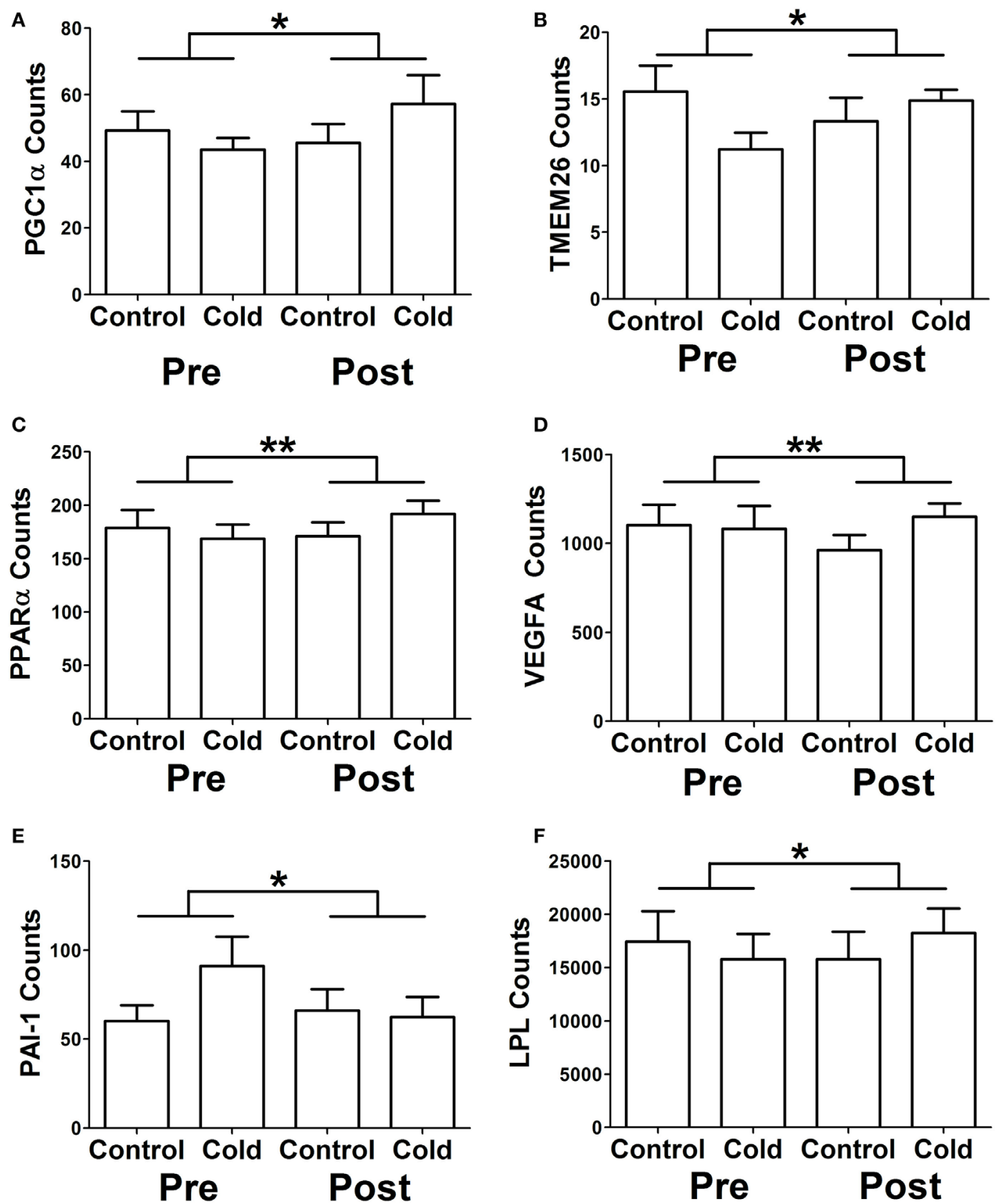

FIGURE 2 | KDT501 treatment alters the subcutaneous white adipose tissue (SC WAT) transcriptional response to cold exposure. The Nanostring nCounter system was used to measure gene expression in SC WAT of subjects treated before and after 1 month KDT501 treatment. A-F) Genes that had a different response to the cold stimulus were identified as described in the Section "Materials and Methods." Data represent the mean \pm SEM $(n=9)$; data in all panels were analyzed by a paired, two tailed Student's $t$-test of the change in gene expression by cold before and after KDT501 treatment $\left({ }^{\star} P<0.05\right.$; $\left.{ }^{* \star} P<0.01\right)$.

Recently, a study by Komai and colleagues found that $\beta$ - adrenergic signaling promotes adiponectin secretion (22). Since cold stimulates the sympathetic nervous system and $\beta$-adrenergic signaling, another potential mechanism for KDT501 stimulation of adiponectin secretion could involve this pathway. To explore this further, we measured adiponectin protein levels from the SC WAT explants and observed that cold-stimulated adiponectin secretion. Cold-stimulated total (Figure 4A; $P<0.01$ ) and HMW adiponectin (Figure 4B; $P<0.01$ ) secretion from the SC WAT explants before KDT501 treatment but did not affect adiponectin mRNA expression (Figure 4C). Cold also stimulated total (Figure 4D; $P<0.05$ ) and HMW adiponectin (Figure 4E; $P<0.01$ ) secretion from the SC WAT explants after KDT501 treatment but did not affect adiponectin mRNA expression (Figure 4F).

Three proteins that promote adiponectin secretion have been identified and could thus act posttranscriptionally to increase 


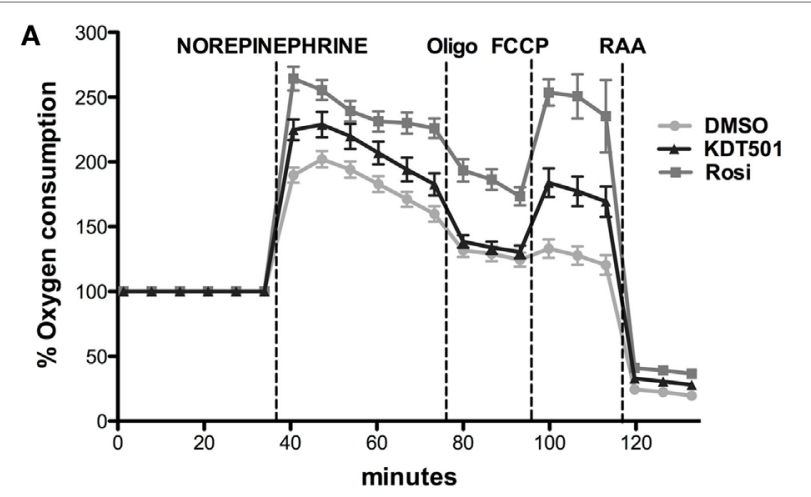

B Basal FAO

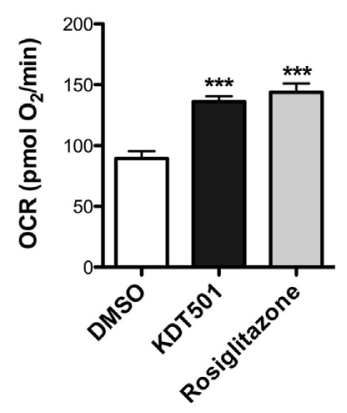

Maximal exogenous 16:0 oxidation

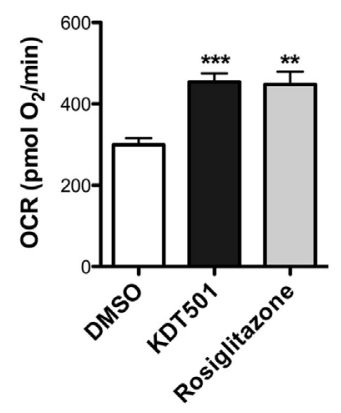

FIGURE 3 | Adipocytes treated with KDT501 show a magnified response to norepinephrine and increased fatty acid oxidation rate. (A) Mouse primary brown adipocytes were treated for $16 \mathrm{~h}$ with DMSO (vehicle), KDT501 $(10 \mu \mathrm{M})$, or rosiglitazone $(2 \mu \mathrm{M})$. Oxygen consumption rate was measured on a Seahorse XFe96 as described in the Section "Materials and Methods." (B) 3T3-L1 adipocytes were treated for $48 \mathrm{~h}$ with KDT501 (10 $\mu \mathrm{M})$ or rosiglitazone $(2 \mu \mathrm{M})$, and the rate of basal (endogenous) and maximal (exogenous palmitate added) fatty acid oxidation measured in real time using the XFe96. Data represent four biological replicates. Error bars indicate SD. ${ }^{\star \star} P<0.01$; ${ }^{\star \star \star} P<0.001$ by one-way analysis of variance.

adiponectin secretion. Because of the increase in adiponectin secretion without any change in mRNA level, we therefore determined whether KDT501 or cold affected the expression of these by real-time RT-PCR (primer sequences are in Table 4). KDT501 treatment alone did not affect the mRNA expression of any of these; however, all three were induced by cold. ERp44 was induced by cold (Figure 5A; pre: $P<0.05$; post: $P<0.001$ ), and KDT501 enhanced this effect (Figure 5A; $P<0.05$ ). Ero-La was induced by cold after KDT501 (Figure 5B; $P<0.05$ ). DsbA-L was induced by cold before and after KDT501 treatment (Figure 5C; pre: $P<0.05$; post: $P<0.01)$.

\section{Summary}

In summary, KDT501 treatment had a beneficial effect on SC WAT of obese human research participants. KDT501 increased adiponectin secretion by a posttranscriptional mechanism and altered the transcriptional response of human adipose tissue to cold stimulation, inducing the expression of many genes involved in thermogenesis and lipolysis. Consistent with these observations, the ability of KDT501 to enhance the response of cultured adipocytes to $\beta$-adrenergic stimuli and increase mitochondrial function provides a potential mechanism of action.

\section{DISCUSSION}

There is a need to develop new therapies to combat obesity and obesity-related disorders such as metabolic syndrome, non-alcoholic fatty liver disease and steatohepatitis, and the lipodystrophy of PCOS. We previously reported that KDT501 treatment of obese, insulin-resistant subjects for 28 days resulted in an increase in plasma adiponectin and HMW adiponectin (2). In this study, we found that SC WAT from these obese, nondiabetic human subjects is a target of KDT501 treatment and investigated the mechanistic basis of its action. KDT501 stimulates total and HMW adiponectin protein secretion from SC WAT explants, and this may be related to the ability of KDT501 to enhance mitochondrial function, which was revealed in experiments using cultured adipocytes. Furthermore, additional analysis uncovered additional effects of KDT501 on SC WAT. Multiplex analysis of gene expression revealed that KDT501 enhanced expression of PGC1 $\alpha, \operatorname{PPAR} \alpha$, and the beige cell marker TMEM26 in response to cold. These findings are consistent with the ability of KDT501 to enhance $\beta$-adrenergic responses in primary brown adipocytes.

Adiponectin exists as a heterogeneous mix of multimers, and plasma HMW adiponectin levels are negatively correlated with various aspects of metabolic syndrome, including insulin resistance (4). Following the transcription of the adiponectin $30 \mathrm{kDa}$ monomer, considerable posttranslational processing and assembly of the HMW multimer occurs, leaving multiple possibilities for regulation. The inability of KDT501 to increase transcription of the adiponectin gene indicates that KDT501 induces adiponectin secretion and multimerization posttranscriptionally. Multimerization of adiponectin into an HMW form and its secretion are complex processes regulated by ER stress, mitochondrial dysfunction, autophagy, and proteins such as ERp44, Ero1-L $\alpha$, and DsbA-L (23-26). Our studies demonstrating that KDT501 enhances fatty acid oxidation in 3T3-L1 adipocytes suggest that KDT501 has beneficial effects on mitochondrial function. Mitochondrial function in adipose tissue is impaired in obesity, and recent studies suggest that impaired mitochondria function reduces adiponectin secretion (27-29). Therefore, the ability of KDT501 to increase adiponectin secretion may be through its ability to enhance mitochondrial function, as was revealed by our in vitro assays. Another possible mechanism for the increase in adiponectin secretion induced by KDT501 could be that KDT501 sensitizes adipocytes to $\beta$-adrenergic agonists, as was revealed by our analysis of SC WAT gene expression and in vitro assays. Recent studies have demonstrated that $\beta$-adrenergic signaling pathways promote adiponectin secretion (22). We found that cold stimulates adiponectin secretion in vivo, suggesting that stimulation of $\beta$-adrenergic signaling increases adiponectin secretion in vivo in obese subjects. Thus, the ability of KDT501 to enhance $\beta$-adrenergic signaling may also contribute to the increased adiponectin secretion.

Pioglitazone has also been shown to induce adiponectin secretion by posttranscriptional mechanisms (30), and PPAR $\gamma$ agonists are known for their powerful effects on increasing adiponectin gene expression (31). However, data from this and other studies 

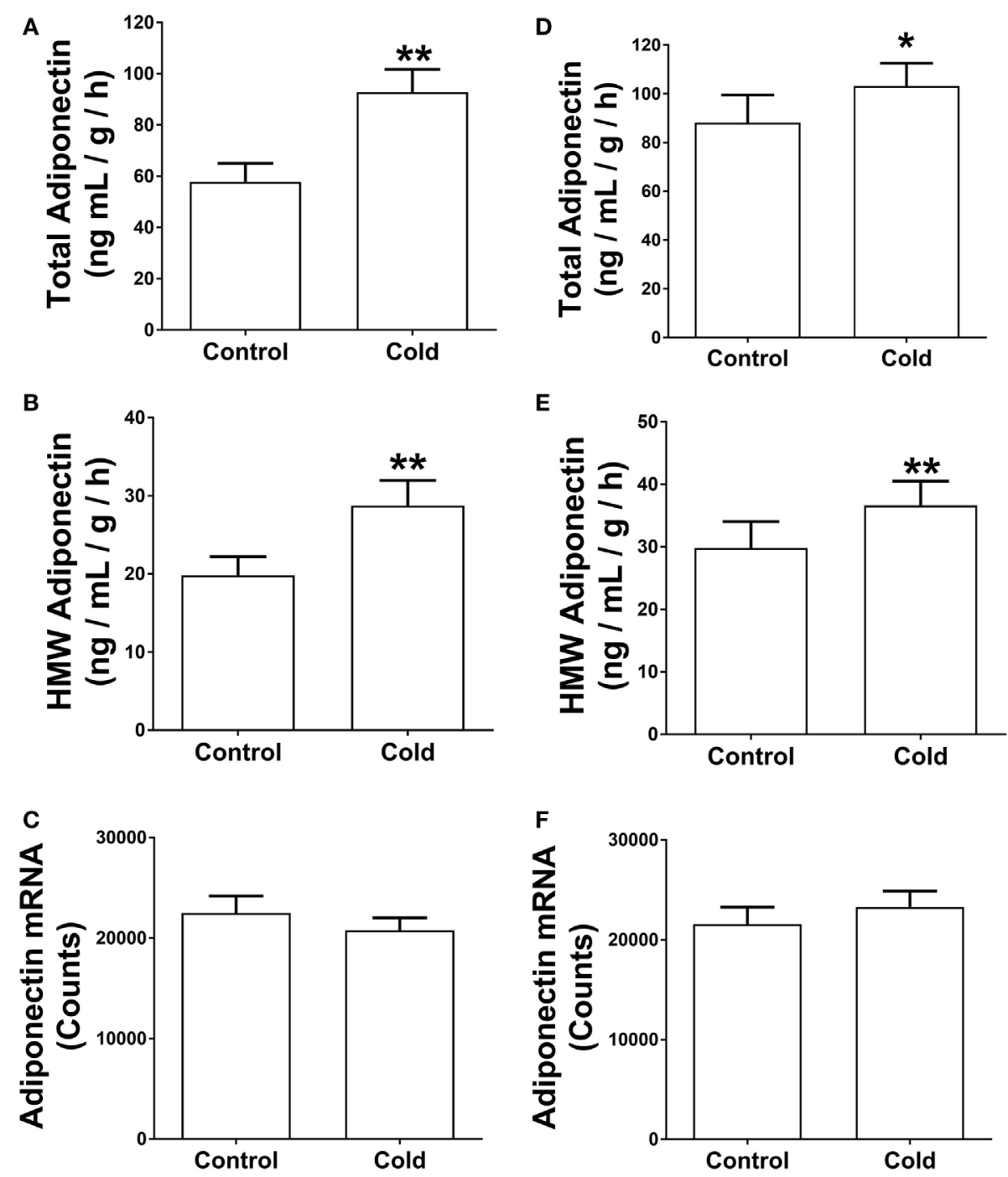

FIGURE 4 | Cold induces total and high-molecular weight (HMW) adiponectin secretion by adipose tissue explants of obese, insulin-resistant subjects. Adipose tissue explants obtained before and after cold treatment (30 min) before and after KDT501 treatment were incubated in Dulbecco's modified Eagles' medium (DMEM) for $4-\mathrm{h}$ at $37^{\circ} \mathrm{C}$. (A) Total and (B) HMW adiponectin secretion in the DMEM were measured by ELISA and are expressed as the concentration/g adipose/h. (C) Adiponectin gene expression was measured in the corresponding adipose tissue biopsy with the Nanostring nCounter system as described in the Section "Materials and Methods." (D) Total and (E) HMW adiponectin secretion in the DMEM were measured by ELISA. (F) Adiponectin gene expression was measured in the corresponding adipose tissue biopsy with the Nanostring nCounter system as described in the Section "Materials and Methods." Data represent the mean \pm SEM $(n=9)$; data in all panels were analyzed by a paired, two tailed Student's $t$-test $\left({ }^{\star} P<0.05 ;{ }^{* \star} P<0.01\right)$.

are not consistent with KDT501 directly stimulating PPAR $\gamma$ transcriptional activity. In vitro, KDT501 has been reported to have pleotropic effects in adipocytes, including suppression of NFKB signaling (1), and, perhaps, a weak PPAR $\gamma$ agonist effect. However, in this study, we found no evidence of KDT501 PPAR $\gamma$ agonism in humans. Direct PPAR $\gamma$ target genes such as adiponectin, LPL, CD36, PNPLA2, and FABP4 were not induced by KDT501 treatment (TableS2 in Supplementary Material). Suppression of inflammation would also be predicted to promote adiponectin gene expression, but numerous inflammatory cytokine and immune cell markers were measured and not affected by KDT501. Thus, it will be important to perform additional studies to determine the mechanism by which KDT501 exerts changes in adiponectin circulating levels. Our in vitro studies suggest that an improvement in mitochondrial function may be an important part of the mechanism.

The data in this report are consistent with the increase in plasma adiponectin in these research participants reported earlier (2). Adipose tissue explants from these same subjects secreted more total and HMW adiponectin after KDT501 treatment. Although adiponectin levels in plasma were increased, our clinical study did not reveal changes in glucose metabolism or insulin sensitivity (2). These subjects were only treated with KDT501 for 28 days, and it is possible that longer treatment or 
TABLE 4 | Real-time reverse transcriptase polymerase chain reaction primers.

\begin{tabular}{lll}
\hline Gene & Forward & Reverse \\
\hline ACTB & GAG CAC AGA GCC & CGC GGC GAT ATC \\
PP1A & TCG CCT TT ATC CAT \\
& CCC ACC GTG TTC & GCT GTC TT GGG \\
PP1B & ATC GAC AT & ACC TTG TCT \\
& AGG TCA CCG TCA ATT TT & TGC TGT Tा TGT \\
TBP & CCC GAA ACG CCG & AGC CAA ATC CT \\
TUBB & AAT ATA ATC C & AAT CAG TGC CGT \\
UBC 9 & ACC AAC CTA CGG & GGT TCG TG \\
& GGA TCT GAA & TTG ACT GCC AAC \\
ERp44 & CTG GAA GAT GGT & TTG CGG A \\
ERO1L & CGT ACC CTG & GGT CT GCC AGT \\
& AGC CCA GAG ATA & GAG TGT CT \\
DsbA-L & CAG GAT AAG C & GT GCC TGA TGT \\
& GGC TGG GGA TTC & AAT CTG CCA \\
\hline & TTG TT GG & AGT AAC CAC TAA \\
& TCT GGA AAA GAT & CCT GGC AGA \\
& CGC AAC GC & GCC CAA AGG CTC \\
& & CGT ATC TG \\
\hline
\end{tabular}

higher blood levels of KDT501 are necessary to realize the full beneficial effects of its ability to increase adiponectin.

Despite increasing the levels of adiponectin, which is an antiinflammatory adipokine, KDT501 had minimal effects on gene expression in the absence of a cold stimulus. One explanation for the lack of effect on gene expression is that the short-term treatment did not cause weight loss (2), change \% body fat, or change resting energy expenditure. KDT501 treatment remodeled the transcriptional response of adipose tissue to cold and increased expression of genes involved in lipolysis and thermogenesis. We have recently reported that lean, healthy subjects respond to the same cold stimulus used in this study with a significant increase in expression of PGC1 $\alpha$ and TMEM26 at the RNA level (13). The induction of PGC1 $\alpha$ and TMEM26 in response to cold after KDT501 treatment seen here was lower than that measured in the lean subjects of our earlier study (13). Nonetheless, this indicates a beneficial effect of KDT501 on SC WAT, for PGC1 $\alpha$ expression in white adipose tissue promotes insulin sensitivity (32). It is also important to note that this study was done entirely in the summer. We have previously documented seasonal effects on thermogenic gene expression in humans. Baseline expression of thermogenic genes in lean subjects is lower in the summer, and we saw greater changes in response to a cold stimulus in the summer (13). These findings indicate reduced $\beta$-adrenergic signaling in humans in the summer, which may explain why greater changes in PGC1 $\alpha$ and TMEM26 expression after KDT501 treatment were not seen in the present obese subjects. KDT501 robustly enhanced NE-stimulated cellular respiration in primary brown adipocytes, suggesting a mechanism for the increase in PGC1 $\alpha$ and TMEM26 expression upon cold exposure in vivo: KDT501 sensitizes adipose tissue to $\beta$-adrenergic signaling. This mechanism may contribute to the weight loss observed in KDT501-treated mice, studies that were performed at room temperature, below thermoneutrality for mice (1).
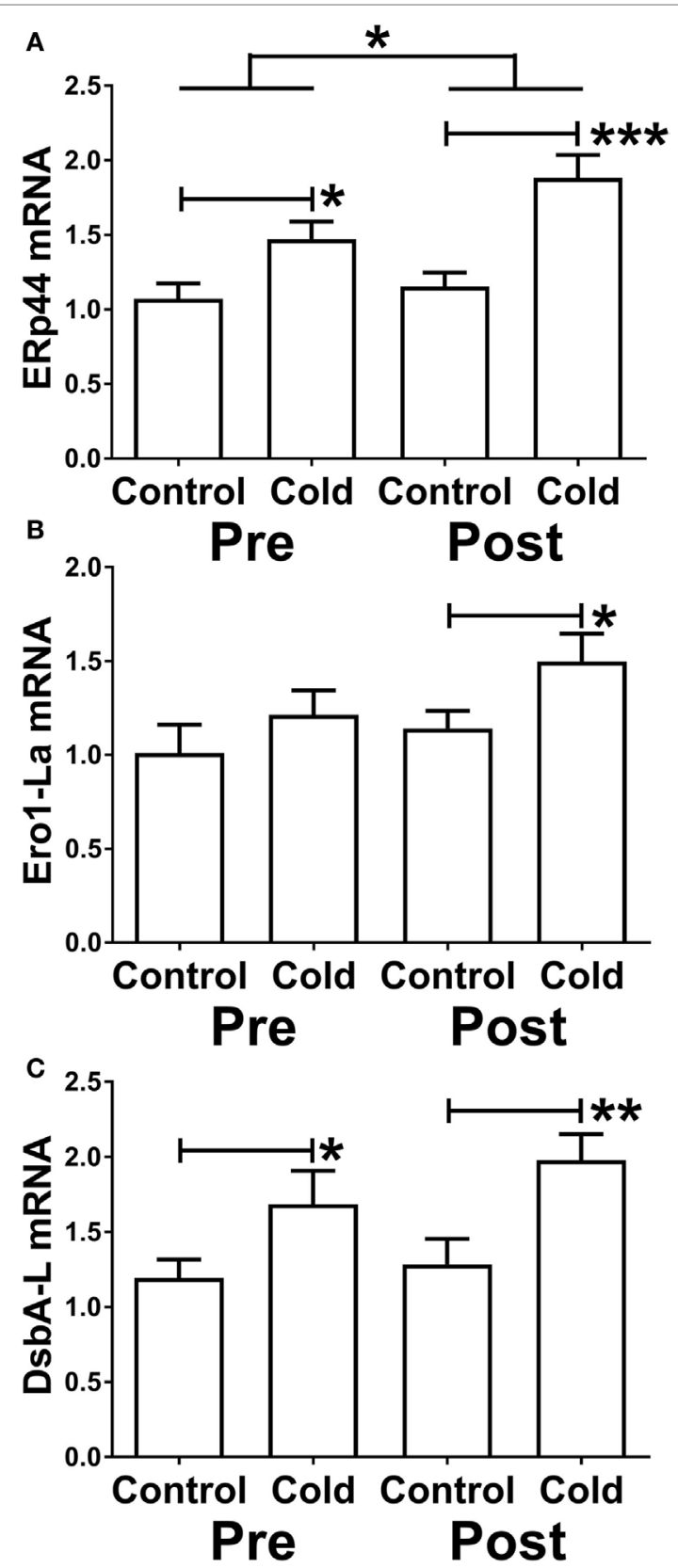

FIGURE 5 | Effect of KDT501 and cold on the expression of genes that regulate adiponectin secretion and multimerization. (A-C) Gene expression was measured by real-time reverse transcriptase polymerase chain reaction as described in the Section "Materials and Methods." Data represent the mean $\pm \operatorname{SEM}(n=9)$; data in all panels were analyzed by a repeated measures analysis of variance $\left({ }^{\star} P<0.05 ;{ }^{\star \star} P<0.01 ;{ }^{\star \star \star} P<0.001\right)$. The data in all panels were also analyzed by a paired, two tailed Student's $t$-test of the change in gene expression by cold before and after KDT501 treatment (ERp44: $\left.{ }^{\star} P<0.05\right)$

ANGPTL4 expression was highly induced by cold after KDT501 treatment. Since ANGPTL4 can be induced by free fatty acids acting through PPAR $\delta$ (33), this may be a sign of increased lipolysis. Interestingly, ANGPTL4 itself contributes to lipolysis by stimulating adipocyte cAMP levels $(34,35)$. Recent studies 
in mice indicate that ANGPTL4 is induced in WAT in response to cold to inhibit LPL and thus favor the delivery of lipids to BAT (36). KDT501 appears to restore this tissue-specific regulation of ANGPTL4 by cold in obese subjects. PGC1 $\alpha$ was also induced by cold after KDT501 treatment along with a number of other genes involved in lipid catabolism, including PPAR $\alpha$. Notably, UCP1 expression was not induced. This finding may be ascribed to the fact that cold-stimulated induction of UCP1 is blunted by chronic inflammation in obese subjects (13), and KDT501 did not reduce SC WAT inflammation, as evidenced by a lack of change in expression of multiple inflammatory genes. It is also possible that the timing of the biopsies, $4 \mathrm{~h}$ after the $30 \mathrm{~min}$ cold stimulus, may not have been suited to capture cold-induced changes in UCP1 mRNA.

Taken together, the results of this initial study reveal that SC WAT is a physiologic target of KDT501. KDT501 acts by at least two mechanisms. It promotes adiponectin secretion by a posttranscriptional mechanism that may be related to its ability to improve mitochondrial function. KDT501 also enhances thermogenic gene expression upon cold exposure, a property that may be due to its ability to sensitize cultured adipocytes to $\beta$-adrenergic stimuli. Longer exposure to KDT501 may have beneficial effects on adipose tissue dysfunction implicated in conditions such as T2D, PCOS, and NASH in humans.

\section{ETHICS STATEMENT}

All subjects gave informed consent, and the protocols were approved by the Institutional Review Board at the University of Kentucky.

\section{REFERENCES}

1. Konda VR, Desai A, Darland G, Grayson N, Bland JS. KDT501, a derivative from hops, normalizes glucose metabolism and body weight in rodent models of diabetes. PLoS One (2014) 9:e87848. doi:10.1371/journal.pone. 0087848

2. Kern PA, Finlin BS, Ross D, Boyechko T, Zhu B, Grayson N, et al. Effects of KDT501 on metabolic parameters in insulin resistant prediabetic humans. J Endocr Soc (2017) 1:650-9. doi:10.1210/js.2017-00202

3. Cankaya S, Demir B, Aksakal SE, Dilbaz B, Demirtas C, Goktolga U. Insulin resistance and its relationship with high molecular weight adiponectin in adolescents with polycystic ovary syndrome and a maternal history of polycystic ovary syndrome. Fertil Steril (2014) 102:826-30. doi:10.1016/j. fertnstert.2014.05.032

4. Stern JH, Rutkowski JM, Scherer PE. Adiponectin, leptin, and fatty acids in the maintenance of metabolic homeostasis through adipose tissue crosstalk. Cell Metab (2016) 23:770-84. doi:10.1016/j.cmet.2016.04.011

5. Hotamisligil GS, Arner P, Caro JF, Atkinson RL, Spiegelman BM. Increased adipose tissue expression of tumor necrosis factor-alpha in human obesity and insulin resistance. J Clin Invest (1995) 95:2409-15. doi:10.1172/ JCI117936

6. Kern PA, Ranganathan S, Li C, Wood L, Ranganathan G. Adipose tissue tumor necrosis factor and interleukin-6 expression in human obesity and insulin resistance. Am J Physiol Endocrinol Metab (2001) 280:E745-51.

7. Kern PA, Di Gregorio GB, Lu T, Rassouli N, Ranganathan G. Adiponectin expression from human adipose tissue: relation to obesity, insulin resistance, and tumor necrosis factor-alpha expression. Diabetes (2003) 52:1779-85. doi:10.2337/diabetes.52.7.1779

8. Kovacova Z, Tencerova M, Roussel B, Wedellova Z, Rossmeislova L, Langin $\mathrm{D}$, et al. The impact of obesity on secretion of adiponectin multimeric

\section{AUTHOR CONTRIBUTIONS}

$\mathrm{PK}, \mathrm{ES}$, and $\mathrm{BF}$ designed the experiments, analyzed data, and wrote the manuscript. BZ, BK, and CG performed the experiments. PW analyzed data. NG, RS, and JB edited the manuscript. $\mathrm{PK}$ is the guarantor of this work, has full access to all of this data, and takes responsibility for it integrity and the accuracy of its analysis.

\section{ACKNOWLEDGMENTS}

The authors wish to thank the staff of the University of Kentucky Clinical Research Unit for the assistance with this study, especially Dr. Jen Moylan for the adiponectin ELISAs, Donna Wall for technical assistance with the nanostring assays, and Dorothy Ross for coordinating the recruitment of the participants.

\section{FUNDING}

This work was supported by Kindex Pharmaceuticals, National Institute of Diabetes and Digestive and Kidney Diseases (DK107646), National Institute of General Medical Sciences (P20GM103527), NIH Office of the Director (1S10OD16357), and National Center for Advancing Translational Sciences (CTSA grant UL1TR001998).

\section{SUPPLEMENTARY MATERIAL}

The Supplementary Material for this article can be found online at http://journal.frontiersin.org/article/10.3389/fendo.2017.00255/ full\#supplementary-material.

isoforms differs in visceral and subcutaneous adipose tissue. Int J Obes (Lond) (2012) 36:1360-5. doi:10.1038/ijo.2011.223

9. Finlin BS, Zhu B, Confides AL, Westgate PM, Harfmann BD, Dupont-Versteegden EE, et al. Mast cells promote seasonal white adipose beiging in humans. Diabetes (2017) 66:1237-46. doi:10.2337/db16-1057

10. Gantner ML, Hazen BC, Conkright J, Kralli A. GADD45gamma regulates the thermogenic capacity of brown adipose tissue. Proc Natl Acad Sci U S A (2014) 111:11870-5. doi:10.1073/pnas.1406638111

11. Dominguez E, Galmozzi A, Chang JW, Hsu KL, Pawlak J, Li W, et al. Integrated phenotypic and activity-based profiling links Ces3 to obesity and diabetes. Nat Chem Biol (2014) 10:113-21. doi:10.1038/nchembio.1429

12. Wu J, Bostrom P, Sparks LM, Ye L, Choi JH, Giang AH, et al. Beige adipocytes are a distinct type of thermogenic fat cell in mouse and human. Cell (2012) 150:366-76. doi:10.1016/j.cell.2012.05.016

13. Kern PA, Finlin BS, Zhu B, Rasouli N, McGehee RE Jr, Westgate PM, et al. The effects of temperature and seasons on subcutaneous white adipose tissue in humans: evidence for thermogenic gene induction. J Clin Endocrinol Metab (2014) 99:E2772-9. doi:10.1210/jc.2014-2440

14. Sun K, Wernstedt Asterholm I, Kusminski CM, Bueno AC, Wang ZV, Pollard JW, et al. Dichotomous effects of VEGF-A on adipose tissue dysfunction. Proc Natl Acad Sci U S A (2012) 109:5874-9. doi:10.1073/pnas. 1200447109

15. Gombert M, Dieu-Nosjean MC, Winterberg F, Bunemann E, Kubitza RC, Da Cunha L, et al. CCL1-CCR8 interactions: an axis mediating the recruitment of $\mathrm{T}$ cells and Langerhans-type dendritic cells to sites of atopic skin inflammation. J Immunol (2005) 174:5082-91. doi:10.4049/jimmunol.174. 8.5082

16. Sironi M, Martinez FO, D’Ambrosio D, Gattorno M, Polentarutti N, Locati M, et al. Differential regulation of chemokine production by Fcgamma receptor engagement in human monocytes: association of CCL1 with a distinct form 
of M2 monocyte activation (M2b, type 2). J Leukoc Biol (2006) 80:342-9. doi:10.1189/jlb.1005586

17. Lee MW, Odegaard JI, Mukundan L, Qiu Y, Molofsky AB, Nussbaum JC, et al. Activated type 2 innate lymphoid cells regulate beige fat biogenesis. Cell (2015) 160:74-87. doi:10.1016/j.cell.2014.12.011

18. Qiu Y, Nguyen KD, Odegaard JI, Cui X, Tian X, Locksley RM, et al. Eosinophils and type 2 cytokine signaling in macrophages orchestrate development of functional beige fat. Cell (2014) 157:1292-308. doi:10.1016/j. cell.2014.03.066

19. Nguyen KD, Qiu Y, Cui X, Goh YP, Mwangi J, David T, et al. Alternatively activated macrophages produce catecholamines to sustain adaptive thermogenesis. Nature (2011) 480:104-8. doi:10.1038/nature10653

20. Rao RR, Long JZ, White JP, Svensson KJ, Lou J, Lokurkar I, et al. Meteorin-like is a hormone that regulates immune-adipose interactions to increase beige fat thermogenesis. Cell (2014) 157:1279-91. doi:10.1016/j.cell.2014.03.065

21. Yadav H, Quijano C, Kamaraju AK, Gavrilova O, Malek R, Chen W, et al. Protection from obesity and diabetes by blockade of TGF-beta/Smad3 signaling. Cell Metab (2011) 14:67-79. doi:10.1016/j.cmet.2011.04.013

22. Komai AM, Musovic S, Peris E, Alrifaiy A, El Hachmane MF, Johansson M, et al. White adipocyte adiponectin exocytosis is stimulated via beta3-adrenergic signaling and activation of Epac1: catecholamine resistance in obesity and type 2 diabetes. Diabetes (2016) 65:3301-13. doi:10.2337/db15-1597

23. Liu M, Liu F. Regulation of adiponectin multimerization, signaling and function. Best Pract Res Clin Endocrinol Metab (2014) 28:25-31. doi:10.1016/j. beem.2013.06.003

24. Zhou L, Liu M, Zhang J, Chen H, Dong LQ, Liu F. DsbA-L alleviates endoplasmic reticulum stress-induced adiponectin downregulation. Diabetes (2010) 59:2809-16. doi:10.2337/db10-0412

25. Wang ZV, Schraw TD, Kim JY, Khan T, Rajala MW, Follenzi A, et al. Secretion of the adipocyte-specific secretory protein adiponectin critically depends on thiol-mediated protein retention. Mol Cell Biol (2007) 27:3716-31. doi:10.1128/MCB.00931-06

26. Qiang L, Wang H, Farmer SR. Adiponectin secretion is regulated by SIRT1 and the endoplasmic reticulum oxidoreductase Ero1-L alpha. Mol Cell Biol (2007) 27:4698-707. doi:10.1128/MCB.02279-06

27. Koh EH, Park JY, Park HS, Jeon MJ, Ryu JW, Kim M, et al. Essential role of mitochondrial function in adiponectin synthesis in adipocytes. Diabetes (2007) 56:2973-81. doi:10.2337/db07-0510

28. Kusminski CM, Scherer PE. Mitochondrial dysfunction in white adipose tissue. Trends Endocrinol Metab (2012) 23:435-43. doi:10.1016/j.tem.2012. 06.004
29. Frizzell N, Rajesh M, Jepson MJ, Nagai R, Carson JA, Thorpe SR, et al. Succination of thiol groups in adipose tissue proteins in diabetes: succination inhibits polymerization and secretion of adiponectin. J Biol Chem (2009) 284:25772-81. doi:10.1074/jbc.M109.019257

30. Bodles AM, Banga A, Rasouli N, Ono F, Kern PA, Owens RJ. Pioglitazone increases secretion of high-molecular-weight adiponectin from adipocytes. Am J Physiol Endocrinol Metab (2006) 291:E1100-5. doi:10.1152/ajpendo. 00187.2006

31. Rasouli N, Yao-Borengasser A, Miles LM, Elbein SC, Kern PA. Increased plasma adiponectin in response to pioglitazone does not result from increased gene expression. Am J Physiol Endocrinol Metab (2006) 290:E42-6. doi:10.1152/ajpendo.00240.2005

32. Kleiner S, Mepani RJ, Laznik D, Ye L, Jurczak MJ, Jornayvaz FR, et al. Development of insulin resistance in mice lacking PGC-1alpha in adipose tissues. Proc Natl Acad Sci U S A (2012) 109:9635-40. doi:10.1073/pnas. 1207287109

33. Staiger H, Haas C, Machann J, Werner R, Weisser M, Schick F, et al. Muscle-derived angiopoietin-like protein 4 is induced by fatty acids via peroxisome proliferator-activated receptor (PPAR)-delta and is of metabolic relevance in humans. Diabetes (2009) 58:579-89. doi:10.2337/db07-1438

34. Gray NE, Lam LN, Yang K, Zhou AY, Koliwad S, Wang JC. Angiopoietinlike 4 (Angptl4) protein is a physiological mediator of intracellular lipolysis in murine adipocytes. J Biol Chem (2012) 287:8444-56. doi:10.1074/jbc. M111.294124

35. Koliwad SK, Gray NE, Wang JC. Angiopoietin-like 4 (Angptl4): a glucocorticoid-dependent gatekeeper of fatty acid flux during fasting. Adipocyte (2012) 1:182-7. doi:10.4161/adip.20787

36. Dijk W, Heine M, Vergnes L, Boon MR, Schaart G, Hesselink MK, et al. ANGPTL4 mediates shuttling of lipid fuel to brown adipose tissue during sustained cold exposure. Elife (2015) 4:e08428. doi:10.7554/eLife.08428

Conflict of Interest Statement: This study was funded by Kindex Pharmaceuticals (Seattle, WA).

Copyright ๔ 2017 Finlin, Zhu, Kok, Godio, Westgate, Grayson, Sims, Bland, Saez and Kern. This is an open-access article distributed under the terms of the Creative Commons Attribution License (CC BY). The use, distribution or reproduction in other forums is permitted, provided the original author $(s)$ or licensor are credited and that the original publication in this journal is cited, in accordance with accepted academic practice. No use, distribution or reproduction is permitted which does not comply with these terms. 